QUARTERLY OF APPLIED MATHEMATICS

VOLUME LXX, NUMBER 1

MARCH 2012, PAGES 77-97

S 0033-569X(2011)01239-2

Article electronically published on September 16, 2011

\title{
LAPLACE TRANSFORM OF PRODUCTS OF BESSEL FUNCTIONS: A VISITATION OF EARLIER FORMULAS
}

\author{
BY \\ EDUARDO KAUSEL (Professor of Civil and Environmental Engineering, Massachusetts Institute of \\ Technology, Cambridge, Massachusetts 02139) \\ AND \\ MIRZA M. IRFAN BAIG (Simpson, Gumpertz \& Heger Inc., Waltham, Massachusetts 02453)
}

\begin{abstract}
This note deals with the Laplace transforms of integrands of the form $x^{\lambda} J_{\alpha}(a x) J_{\beta}(b x)$, which are found in numerous fields of application. Specifically, we provide herein both a correction and a supplement to the list of integrals given in 1997 by Hanson and Puja, who in turn extended the formulas of Eason, Noble and Sneddon of 1956. The paper concludes with an extensive tabulation for particular cases and range of parameters.
\end{abstract}

1. Introduction. In a classic 1956 paper, Eason, Noble and Sneddon - henceforth ENS for short - presented a general methodology for finding integrals involving products of Bessel functions, and provided a set of closed-form formulas for cases commonly encountered in engineering science and in applied mathematics. Although these integrals extended considerably the repertoire of exact formulas available in standard tables such as Oberhettinger's or Gradshteyn and Ryzhik's, even to this day programs such as Mathematica, Maple and Matlab's symbolic tool seem to have remained unaware of the ENS paper, for they are unable to provide answers to such integrals. Some four decades later, Hanson and Puja (1997) - henceforth denoted as HP - reconsidered the ENS paper and not only extended considerably the formulas therein, but by changing the arguments to the functions, they arrived at alternative forms which allegedly avoided discontinuities at certain values of the parameters. Unfortunately, once the arguments in the HP formulas exceed some threshold value, the computations for some of the integrals suffer a complete breakdown and become useless. This led us to investigate both the reasons for the erroneous results and also to seek a corrected set of formulas, which constitutes the subject of this paper.

Received June 2, 2010.

2010 Mathematics Subject Classification. Primary 44-00, 44A10, 74-00.

E-mail address: kausel@mit.edu

E-mail address: irfan.baig@gmail.com

(C)2011 Brown University

Reverts to public domain 28 years from publication 
To avoid repetitions, the presentation herein will be rather terse, avoiding needless explanations of well-known facts and/or of details which can be found in the originals of the papers referred to. Also, to distinguish clearly between the equations and parameters used by ENS and HP, we have adopted a revised, more general notation. We defer a description of the problem itself until section 5 so as to summarize first some needed preliminary definitions and properties.

2. Laplace transform and its properties. The integrals in question are the Laplace transforms

$$
I_{\alpha \beta}^{\lambda} \equiv I_{\alpha \beta}^{\lambda}(a, b, s)=\int_{0}^{\infty} x^{\lambda} J_{\alpha}(a x) J_{\beta}(b x) e^{-s x} d x
$$

with

$$
\begin{aligned}
& \alpha+\beta+\lambda>-1 \text { if } s>0, \\
& \alpha+\beta+1>-\lambda>-1 \text { if } s=0 \quad \& \quad a \neq b, \\
& \alpha+\beta+1>-\lambda>0 \text { if } s=0 \quad \& \quad a=b,
\end{aligned}
$$

where the parameters $\alpha, \beta, \lambda$ are assumed to be integers and the arguments $a, b, s$ are real. These integrals satisfy the following symmetries and recursive properties:

$$
\begin{array}{cl}
I_{\alpha \beta}^{\lambda}(a, b, s)=I_{\beta \alpha}^{\lambda}(b, a, s), & I_{\alpha \beta}^{\lambda}(a, b, s)=\left\{\begin{array}{c}
\left(\frac{1}{b}\right)^{\lambda+1} I_{\alpha \beta}^{\lambda}\left(\frac{a}{b}, 1, \frac{s}{b}\right), \\
\left(\frac{1}{a}\right)^{\lambda+1} I_{\alpha \beta}^{\lambda}\left(1, \frac{b}{a}, \frac{s}{a}\right),
\end{array}\right. \\
\alpha I_{\alpha \beta}^{\lambda}=\frac{1}{2} a\left(I_{\alpha-1, \beta}^{\lambda+1}+I_{\alpha+1, \beta}^{\lambda+1}\right), & \beta I_{\alpha \beta}^{\lambda}=\frac{1}{2} b\left(I_{\alpha, \beta-1}^{\lambda+1}+I_{\alpha, \beta+1}^{\lambda+1}\right), \\
\frac{\partial I_{\alpha \beta}^{\lambda}}{\partial a}=\frac{1}{2}\left(I_{\alpha-1, \beta}^{\lambda+1}-I_{\alpha+1, \beta}^{\lambda+1}\right), & \frac{\partial I_{\alpha \beta}^{\lambda}}{\partial b}=\frac{1}{2}\left(I_{\alpha, \beta-1}^{\lambda+1}-I_{\alpha, \beta+1}^{\lambda+1}\right) .
\end{array}
$$

A combination of two of the above yields

$$
\begin{aligned}
\frac{\partial I_{\alpha \beta}^{\lambda}}{\partial a} & =I_{\alpha-1, \beta}^{\lambda+1}-\frac{\alpha}{a} I_{\alpha \beta}^{\lambda}, & \frac{\partial I_{\alpha \beta}^{\lambda}}{\partial b} & =I_{\alpha, \beta-1}^{\lambda+1}-\frac{\beta}{b} I_{\alpha \beta}^{\lambda}, \\
& =\frac{\alpha}{a} I_{\alpha \beta}^{\lambda}-I_{\alpha+1, \beta}^{\lambda+1}, & & =\frac{\beta}{b} I_{\alpha \beta}^{\lambda}-I_{\alpha, \beta+1}^{\lambda+1} .
\end{aligned}
$$

In addition,

$$
I_{\alpha \beta}^{\lambda}=-\frac{\partial I_{\alpha \beta}^{\lambda-1}}{\partial s}
$$

It can also be shown via integration by parts that

$$
\begin{aligned}
& (\alpha+\beta-\lambda) I_{\alpha \beta}^{\lambda-1}=a I_{\alpha-1, \beta}^{\lambda}+b I_{\alpha, \beta-1}^{\lambda}-s I_{\alpha \beta}^{\lambda}+\left[x^{\lambda} J_{\alpha}(a x) J_{\beta}(b x)\right]_{x=0}, \\
& \lambda I_{\alpha \beta}^{\lambda-1}=a \frac{1}{2}\left(I_{\alpha+1, \beta}^{\lambda}-I_{\alpha-1, \beta}^{\lambda}\right)+b \frac{1}{2}\left(I_{\alpha, \beta+1}^{\lambda}-I_{\alpha, \beta-1}^{\lambda}\right)+s I_{\alpha \beta}^{\lambda}-\left[x^{\lambda} J_{\alpha}(a x) J_{\beta}(b x)\right]_{x=0}
\end{aligned}
$$

provided $\alpha+\beta+\lambda \geq 0$. The boundary term at $x=0$ vanishes if $\alpha+\beta+\lambda>0$. For example, if $\lambda=0, \alpha=\beta=1$, then

$$
\begin{gathered}
I_{11}^{-1}=\frac{1}{2}\left(a I_{0,1}^{0}+b I_{1,0}^{0}-s I_{11}^{0}\right), \\
0=\frac{1}{2} a\left(I_{2,1}^{0}-I_{0,1}^{0}\right)+\frac{1}{2} b\left(I_{1,2}^{0}-I_{1,0}^{0}\right)+s I_{1,1}^{0},
\end{gathered}
$$


both of which can be useful.

3. Definitions, parameters and fundamental relations.

$$
\begin{aligned}
& A=A(a, b, s)=\sqrt{(a+b)^{2}+s^{2}}, \quad B=B(a, b, s)=\sqrt{(a-b)^{2}+s^{2}}, \\
& L_{1}=L_{1}(a, b, s)=\frac{1}{2}(A-B), \quad L_{2}=L_{2}(a, b, s)=\frac{1}{2}(A+B), \\
& \kappa=\frac{2 \sqrt{L_{1} L_{2}}}{L_{1}+L_{2}}=\frac{2 \sqrt{a b}}{\sqrt{(a+b)^{2}+s^{2}}}=\frac{2 \sqrt{k}}{1+k} \\
& \kappa^{\prime}=\sqrt{1-\kappa^{2}}=\frac{B}{A}=\frac{\sqrt{(a-b)^{2}+s^{2}}}{\sqrt{(a+b)^{2}+s^{2}}}=\frac{1-k}{1+k} \\
& k=\frac{L_{1}}{L_{2}}=\frac{a b}{L_{2}^{2}}=\frac{L_{1}^{2}}{a b}=\frac{1-\kappa^{\prime}}{1+\kappa^{\prime}} \\
& k^{\prime}=\sqrt{1-k^{2}}=\frac{2 \sqrt{A B}}{A+B}=\frac{2 \sqrt{\kappa^{\prime}}}{1+\kappa^{\prime}} \\
& \nu=\frac{4 a b}{(a+b)^{2}}, n_{a b}=\frac{L_{1}^{2}}{b^{2}}=\frac{a^{2}}{L_{2}^{2}}=\frac{a}{b} \frac{L_{1}}{L_{2}}=k \frac{a}{b}, n_{b a}=\frac{L_{1}^{2}}{a^{2}}=\frac{b^{2}}{L_{2}^{2}}=\frac{b}{a} \frac{L_{1}}{L_{2}}=k \frac{b}{a}
\end{aligned}
$$

with

$$
\kappa^{2}<\nu<1, \quad k^{2}<n_{a b}<1, \quad k^{2}<n_{b a}<1, \quad n_{a b} n_{b a}=k^{2} .
$$

These parameters satisfy the following useful relationships:

$$
\begin{gathered}
\sqrt{\nu-\kappa^{2}}=\frac{s \kappa}{a+b}, \quad \sqrt{1-\nu}=\frac{|a-b|}{a+b}, \quad \sqrt{\frac{(1-\nu)\left(\nu-\kappa^{2}\right)}{\nu}}=\frac{|a-b|}{a+b} \frac{s}{L_{2}(1+k)}, \\
L_{1}=\frac{1}{2}\left(1-\kappa^{\prime}\right) \sqrt{(a+b)^{2}+s^{2}} \quad L_{2}=\frac{1}{2}\left(1+\kappa^{\prime}\right) \sqrt{(a+b)^{2}+s^{2}} \\
=\frac{1-\kappa^{\prime}}{\kappa} \sqrt{a b}, \quad=\frac{1+\kappa^{\prime}}{\kappa} \sqrt{a b} \\
\left(L_{1}+L_{2}\right)^{2}=(a+b)^{2}+s^{2}, \quad\left(L_{2}-L_{1}\right)^{2}=(a-b)^{2}+s^{2}, \\
L_{1}^{2}+L_{2}^{2}=\frac{1}{2}\left(A^{2}+B^{2}\right)=a^{2}+b^{2}+s^{2}, \quad L_{2}^{2}-L_{1}^{2}=A B=L_{2}^{2}\left(1-k^{2}\right)=4 a b \frac{\kappa^{\prime}}{\kappa^{2}}, \\
k a-b=\frac{s^{2} k a}{L_{1}^{2}-a^{2}}=\frac{s^{2} b}{b^{2}-L_{2}^{2}}, \quad \frac{s^{2}}{L^{2}-L_{1}^{2}}=\frac{1}{n_{a b}}-1, \quad \frac{s^{2}}{L_{2}^{2}-a^{2}}=1-n_{b a}, \\
\frac{s^{2} k b}{L_{1}^{2}-b^{2}}=\frac{s^{2} a}{a^{2}-L_{2}^{2}}, \quad \frac{s^{2}}{b^{2}-L_{1}^{2}}=\frac{1}{n_{b a}}-1, \quad \frac{s^{2}}{L_{2}^{2}-b^{2}}=1-n_{a b} .
\end{gathered}
$$


4. Elliptic integrals. All of the transforms considered in this work result in formulas involving the complete elliptic integrals of the first, second and third kind. In addition, ENS introduce a $\Lambda$ function which coincides with Heuman's Lambda Naught function multiplied by $\frac{1}{2} \pi$. In the ensuing, we use $\kappa, \nu$ for the ENS arguments and $k, n$ for the HP arguments to the elliptic integrals. These functions and their mathematical properties are:

$$
\begin{aligned}
& \mathbf{K}(\kappa)=\int_{0}^{1} \frac{d x}{\sqrt{1-x^{2}} \sqrt{1-\kappa^{2} x^{2}}} \\
& =\int_{0}^{\pi / 2} \frac{d \theta}{\sqrt{1-\kappa^{2} \sin ^{2} \theta}} \\
& \mathbf{E}(\kappa)=\int_{0}^{1} \frac{\sqrt{1-\kappa^{2} x^{2}} d x}{\sqrt{1-x^{2}}} \\
& =\int_{0}^{\pi / 2} \sqrt{1-\kappa^{2} \sin ^{2} \theta} d \theta \text {, } \\
& \boldsymbol{\Pi}(\nu, \kappa)=\int_{0}^{1} \frac{d x}{\left(1-\nu x^{2}\right) \sqrt{1-x^{2}} \sqrt{1-\kappa^{2} x^{2}}} \\
& =\int_{0}^{\pi / 2} \frac{d \theta}{\left(1-\nu \sin ^{2} \theta\right) \sqrt{1-\kappa^{2} \sin ^{2} \theta}}, \\
& \Lambda(\nu, \kappa)=\frac{\sqrt{(1-\nu)\left(\nu-\kappa^{2}\right)}}{\sqrt{\nu}} \boldsymbol{\Pi}(\nu, \kappa) \equiv \frac{\pi}{2} \Lambda_{0}(\psi \backslash \vartheta) \\
& =[\mathbf{E}(\kappa)-\mathbf{K}(\kappa)] F\left(\psi \backslash \vartheta^{\prime}\right)+\mathbf{K}(\kappa) E\left(\psi \backslash \vartheta^{\prime}\right),
\end{aligned}
$$

where $\Lambda_{0}$ is Heuman's function, $F$ and $E$ are the incomplete elliptic integrals of the first and second kind, respectively, and both $\vartheta=\arcsin \kappa$ and $\psi$ are defined by the unnumbered equations above ENS-3.8:

$$
\sin ^{2} \psi=\frac{\nu-\kappa^{2}}{\nu \kappa^{\prime 2}}, \quad \cos ^{2} \psi=\frac{\kappa^{2}(1-\nu)}{\nu \kappa^{\prime 2}}
$$

We also define

$$
\boldsymbol{\Pi}_{a b} \equiv \boldsymbol{\Pi}\left(n_{a b}, k\right), \quad \boldsymbol{\Pi}_{b a} \equiv \boldsymbol{\Pi}\left(n_{b a}, k\right)
$$

with $n_{a b}, n_{b a}$ given by (3.7).

\section{Landen transformation:}

$$
\begin{aligned}
& k=\frac{1-\kappa^{\prime}}{1+\kappa^{\prime}}, \quad \mathbf{K}(k)=\frac{1}{2}\left(1+\kappa^{\prime}\right) \mathbf{K}(\kappa), \quad \mathbf{E}(k)=\frac{1}{1+\kappa^{\prime}}\left[\mathbf{E}(\kappa)+\kappa^{\prime} \mathbf{K}(\kappa)\right], \\
& \kappa=\frac{2 \sqrt{k}}{1+k}, \quad \mathbf{K}(\kappa)=(1+k) \mathbf{K}(k), \quad \mathbf{E}(\kappa)=\frac{2}{1+k} \mathbf{E}(k)-(1-k) \mathbf{K}(k) .
\end{aligned}
$$


In Appendix A we also show that

$$
\begin{aligned}
\Lambda(\nu, \kappa) & =\frac{s}{L_{2}}\left[2 \boldsymbol{\Pi}\left(n_{a b}, k\right)-\mathbf{K}(k)\right] \quad a<b \\
& =\frac{s}{L_{2}}\left[2 \boldsymbol{\Pi}\left(n_{b a}, k\right)-\mathbf{K}(k)\right] \quad a>b .
\end{aligned}
$$

Caveat: Users of advanced software such as Mathematica, Maple and Matlab should beware the arguments being used by the elliptic functions in these programs, for the manuals are not crystal clear about the matter. Specifically, in Mathematica the functions EllipticK and EllipticE use $m=k^{2}$ as an argument, i.e. the so-called parameter, while the same-named functions in Maple and in Matlab's symbolic tool use instead the modulus $k$. To add to the confusion, Matlab's intrinsic numerical function ellipke also uses the parameter $m$ instead of the modulus $k$ and restricts it to be real and less than unity. In the case of multiple solutions for the Laplace transforms, Matlab often gives only one of these, and also remains silent about some of the underlying assumptions (e.g. $a<b=1$ and so forth).

5. Description of the problem. Consider the particular case of the transform $I_{11}^{-1}$. From ENS-4.9, this integral is

$$
\begin{aligned}
I_{11}^{-1}=\frac{s}{\pi \sqrt{a b}}\left[\frac{1}{\kappa} \mathbf{E}(\kappa)-\frac{\kappa}{2 a b}\left(a^{2}+b^{2}+\frac{1}{2} s^{2}\right) \mathbf{K}(\kappa)\right] & +\frac{a^{2}-b^{2}}{2 \pi a b} \operatorname{sgn}(a-b) \Lambda(\nu, \kappa) \\
& +\frac{1}{2} \begin{cases}\frac{b}{a} & a>b, \\
1 & a=b, \\
\frac{a}{b} & a<b,\end{cases}
\end{aligned}
$$

which can be written compactly as

$$
\begin{aligned}
I_{11}^{-1}= & \frac{s}{\pi \sqrt{a b}}\left[\frac{1}{\kappa} \mathbf{E}(\kappa)-\frac{\kappa}{2 a b}\left(a^{2}+b^{2}+\frac{1}{2} s^{2}\right) \mathbf{K}(\kappa)\right] \\
& +\frac{1}{4 a b}\left\{a^{2}+b^{2}+\left(a^{2}-b^{2}\right) \operatorname{sgn}(a-b)\left[\frac{2}{\pi} \Lambda(\nu, \kappa)-1\right]\right\}
\end{aligned}
$$

whereas from HP-25, this same integral is

$$
I_{11}^{-1}=\frac{a}{2 b}+\frac{s}{\pi a b L_{2}}\left[L_{2}^{2} \mathbf{E}(k)-\left(L_{2}^{2}+b^{2}\right) \mathbf{K}(k)-\left(a^{2}-b^{2}\right) \mathbf{\Pi}(n, k)\right],
$$

the characteristic $n$ of which coincides with our $n_{a b}$ in (3.7). Observe that HP-25 consists of a single expression for the transform whatever the relative values of $a, b$, and also uses different arguments and coefficients for the elliptic integrals, while ENS-4.9 exhibits an intrinsic discontinuity at the transition $a=b$ for all values of $s$. Regrettably, although the HP expression is indeed continuous, it is not applicable and fails when $a>b$, as will be seen.

Now, HP developed their formulas in the context of a problem in the theory of elasticity concerning a circular load (i.e. "patch") applied onto an elastic half-space and thus they argued for the continuity of the functions on physical grounds. Quoting HP (square brackets are ours): 
It is apparent that when the integral evaluation does not contain Heuman's Lambda function, only one expression is needed to evaluate the integral for all values of the parameters. However, when the integral evaluation also contains Heuman's Lambda function, two different expressions are given for the integral depending on the relative values of $[a, b]$. Since the elastic field inside the half-space is continuous and has continuous derivatives, it is troublesome that the expressions are different depending on being inside or outside the radius of loading. Intuition would lead one to expect a single expression which is valid at every point in the half-space.

Unfortunately, this appeal to physical continuity did not guarantee that the replacement formulas proposed by HP would be correct, for they actually provide spurious results when applied for $a>b$. As it turns out, two separate formulas are needed for the intervals $a<b$ and $a>b$ which, although distinct, maintain the continuity of the solution and its derivative at $a=b$. In the ensuing, we establish the correct mathematical connection between the ENS and HP formulas and provide an extended set of formulas which are free from this problem.

6. Relationship between ENS and HP. HP provide a transformation formula HP-24 between the complete elliptic integral of the third kind with arguments $\nu, \kappa$ and the elliptic integrals with arguments $n, k$. In our current notation and after some simple transformations, their transformation formula would read

$$
\boldsymbol{\Pi}(\nu, \kappa)=\frac{(1+k)(a+b)}{a-b}\left\{\frac{\pi L_{2}}{s} H(a-b)+\mathbf{K}(k)-2 \boldsymbol{\Pi}(n, k)\right\},
$$

which can also be written as

$$
\operatorname{sgn}(a-b) \Lambda(\nu, \kappa)=\pi H(a-b)+\frac{s}{L_{2}}[\mathbf{K}(k)-2 \boldsymbol{\Pi}(n, k)],
$$

where $H(a-b)$ is the Heaviside function, and $n \equiv n_{a b}$. They state that the proof of this formula is contained in an earlier paper of theirs, but we have been unable to locate any such derivation in said paper. Furthermore, that equation holds no obvious connection to the Landen-Gauss transformation 163.02 given on page 39 in Byrd and Friedman (1971), which appears to be restricted to the "hyperbolic" case $n<k^{2}$ and thus excludes the "circular" case at hand $k^{2}<n$. For this reason, we provide a new proof of the HP translation formula in Appendix A, and in the process show that another formula is needed when $a>b$. With reference to Appendix A, the actual transformation formulas are:

$$
\begin{aligned}
& \Lambda(\nu, \kappa)=\frac{s}{L_{2}}\left(2 \boldsymbol{\Pi}_{a b}-\mathbf{K}(k)\right) \quad a<b \\
& \Lambda(\nu, \kappa)=\frac{s}{L_{2}}\left(2 \boldsymbol{\Pi}_{b a}-\mathbf{K}(k)\right) \quad a>b
\end{aligned}
$$


As also demonstrated in Appendix A, $\boldsymbol{\Pi}_{a b}$ and $\boldsymbol{\Pi}_{b a}$ satisfy the relationship

$$
\boldsymbol{\Pi}_{a b}+\boldsymbol{\Pi}_{b a}-\mathbf{K}=\frac{\pi L_{2}}{2 s}
$$

Taking into account this property, the two transformation formulas $(6.3 \mathrm{a}, \mathrm{b})$ can be combined into a single expression by means of Heaviside functions as follows:

$$
\begin{aligned}
\Lambda(\psi, \kappa) & =\frac{s}{L_{2}}\left\{2 \boldsymbol{\Pi}_{a b}[1-H(a-b)]+2 \boldsymbol{\Pi}_{b a} H(a-b)-\mathbf{K}\right\} \\
& =\frac{s}{L_{2}}\left\{2 \boldsymbol{\Pi}_{a b}[1-H(a-b)]+2\left[\frac{\pi L_{2}}{2 s}-\boldsymbol{\Pi}_{a b}+\mathbf{K}\right] H(a-b)-\mathbf{K}\right\} \\
& =\pi H(a-b)+\operatorname{sgn}(a-b) \frac{s}{L_{2}}\left(\mathbf{K}-2 \boldsymbol{\Pi}_{a b}\right),
\end{aligned}
$$

so

$$
\operatorname{sgn}(a-b) \Lambda(\psi, \kappa)=\pi H(a-b)+\frac{s}{L_{2}}\left(\mathbf{K}-2 \mathbf{\Pi}_{a b}\right),
$$

which does coincide with HP's translation formula (6.2). Although this shows (6.2) to be technically correct, HP's integral transform formulas are still incorrect when $a>b$. This is because in their final expressions for the integrals, they failed to include the Heaviside term, which is absent when $a<b$, and simply assumed that the resulting formulas for that case would be valid throughout; i.e., they reasoned that the formulas had to be "continuous". In addition, $\boldsymbol{\Pi}_{a b}$ is ill-conditioned when $a>b$. In retrospect, it seems peculiar that HP should have argued against the discontinuous Lambda function in ENS, only to replace it with yet another discontinuous function and then "forgot" or proceeded to deliberately ignore - this very discontinuity.

Fortunately, to circumvent this problem, it suffices to make use of (6.4) and replace $\boldsymbol{\Pi}_{a b}$ in all HP formulas by $\boldsymbol{\Pi}_{a b}=\mathbf{K}(k)-\boldsymbol{\Pi}_{b a}+\frac{\pi}{2 s} L_{2}$ whenever $a>b$. This is possible because for all $a, b, s>0, k<1, n_{a b}<1, n_{b a}<1$, in which case (6.4) has no singularities and is a continuous function of the ratio $a / b$, even if (6.4) itself remains intrinsically ill-conditioned because either $\boldsymbol{\Pi}_{a b}$ or $\boldsymbol{\Pi}_{b a}$ attains large values, especially when $s$ is small. This is because for $a>b, n_{a b} \rightarrow 1$, and $\boldsymbol{\Pi}_{a b} \rightarrow \infty$, and vice versa for $\boldsymbol{\Pi}_{b a}$.

To demonstrate the application of the preceding transformation formulas, substitute (6.3a) into the ENS form (5.1), and consider also the equivalences 4.9, which yields

$$
\begin{aligned}
I_{11}^{-1}= & \frac{s}{\pi \sqrt{a b}}\left\{\frac{1+\kappa^{\prime}}{\kappa} \mathbf{E}(k)-\frac{2}{\kappa\left(1+\kappa^{\prime}\right)}\left[\kappa^{\prime}+\frac{\kappa^{2}}{4 a b}\left(2 a^{2}+2 b^{2}+s^{2}\right)\right] \mathbf{K}(k)\right\} \\
& +\frac{s}{\pi L_{2}} \frac{a^{2}-b^{2}}{2 a b}\left(\mathbf{K}(k)-2 \boldsymbol{\Pi}_{a b}\right)+\frac{a}{2 b} .
\end{aligned}
$$

Using the definitions for $k, \kappa$ and other parameters in section 3 , it can be shown that

$$
\frac{2}{\kappa\left(1+\kappa^{\prime}\right)}\left[\kappa^{\prime}+\frac{\kappa^{2}}{4 a b}\left(2 a^{2}+2 b^{2}+s^{2}\right)\right]=\frac{1}{\sqrt{a b} L_{2}}\left[L_{2}^{2}+\frac{1}{2}\left(a^{2}+b^{2}\right)\right] .
$$

Hence

$$
I_{11}^{-1}=\frac{s}{\pi a b L_{2}}\left[L_{2}^{2} \mathbf{E}(k)-\left(L_{2}^{2}+b^{2}\right) \mathbf{K}(k)-\left(a^{2}-b^{2}\right) \mathbf{\Pi}_{a b}\right]+\frac{a}{2 b}, \quad a<b,
$$


which coincides with HP-25 (i.e. (5.2)). On the other hand, making use of (6.3b) instead, we obtain

$$
I_{11}^{-1}=\frac{s}{\pi a b L_{2}}\left[L_{2}^{2} \mathbf{E}(k)-\left(L_{2}^{2}+a^{2}\right) \mathbf{K}(k)-\left(b^{2}-a^{2}\right) \mathbf{\Pi}_{b a}\right]+\frac{b}{2 a}, \quad a>b,
$$

which agrees perfectly with the formula that would be obtained by simply exchanging $a, b$ in (6.9), which must hold because of the symmetry of $I_{11}^{-1}$ with respect to the Bessel indices. Finally, if we substitute $\boldsymbol{\Pi}_{a b}=\mathbf{K}(k)-\boldsymbol{\Pi}_{b a}+\frac{\pi}{2 s} L_{2}$ into (6.9), we once more recover (6.10). Thus, this shows that all is consistent.

7. Tables of integrals. Although many of the integrals listed in the pages that follow are directly based on the HP and ENS papers, we have seen fit to simplify these expressions to the extent possible, using for this purpose the very useful equivalences and properties given in section 3. Hence, they do not quite look like those in HP. This also meant that each and every formula had to be carefully checked for errors, including tests against direct numerical integration. Also, since Laplace transforms constitute improper integrals, it was necessary for us to supplement our numerical quadrature with formulas for integrating the tails, a task that is presented in Appendix C.

We have made an utmost effort in avoiding mistakes in both the transcription and proofreading of the typeset document, and believe the formulas to be free from error. Still, readers are strongly encouraged to carefully verify their own personal implementation of these formulas, not only to avoid any remaining, hidden errors, but also to avoid errors that could have crept in during implementation of the formulas from the published paper.

In addition, some of the integrals could be verified against tabulations such as Oberhettinger's and against Matlab symbolic tools, and we identify the formulas thus checked. In addition, we have also used the recurrence relations to verify some (but not all) of the integrals. The reason is that although the recurrence relations involve differentiations and can in principle be used to reduce the expressions to known integrals, the process can be very tedious because of the complexity of the derivatives of the elliptic functions. Indeed, even when the operations are carried out with a computer, say with Matlab, it is often difficult to collect and factor terms in the resulting expressions, and thus, to reduce the formulas into a recognizable form.

Finally, because many of the formulas are discontinuous when either $s \rightarrow 0$ or $a=b$, we have seen fit to provide separate tables for these cases. By and large, the tables list the integrals in descending value of $\lambda$ and in increasing order of the Bessel functions. 
Table 1: Integrals for $\alpha=0, a=0, s>0$. All of the formulas in this section have been obtained with Matlab's symbolic tool.

$$
\begin{array}{rlrl}
L_{2} & =\sqrt{b^{2}+s^{2}}, & \\
I_{0 \beta}^{0}(0, b, s) & =\int_{0}^{\infty} e^{-s x} J_{\beta}(b x) d x=\frac{1}{L_{2}}\left(\frac{b}{L_{2}+s}\right)^{\beta}, & \beta=0,1,2, \ldots, \\
I_{0 \beta}^{-1}(0, b, s) & =\int_{0}^{\infty} e^{-s x} \frac{J_{\beta}(b x)}{x} d x=\frac{1}{\beta}\left(\frac{b}{L_{2}+s}\right)^{\beta}, & \beta=1,2,3, \ldots, \\
I_{0 \beta}^{-2}(0, b, s) & =\int_{0}^{\infty} e^{-s x} \frac{J_{\beta}(b x)}{x^{2}} d x=\frac{\beta L_{2}+s}{\beta\left(\beta^{2}-1\right)}\left(\frac{b}{L_{2}+s}\right)^{\beta}, & & \beta=2,3,4, \ldots, \\
I_{0 \beta}^{-3}(0, b, s) & =\int_{0}^{\infty} e^{-s x} \frac{J_{\beta}(b x)}{x^{3}} d x & & \beta=3,4,5, \ldots \\
& =\frac{\left[\left(\beta^{2}-1\right) L_{2}^{2}+3 s\left(\beta L_{2}+s\right)\right]}{\beta\left(\beta^{2}-1\right)\left(\beta^{2}-4\right)}\left(\frac{b}{L_{2}+s}\right)^{\beta}, &
\end{array}
$$

Table 2: Integrals for $s=0, a>0, b>0$, in dual format.

$$
\begin{aligned}
& \kappa=\frac{2 \sqrt{a b}}{a+b} \leq 1, \quad k= \begin{cases}\frac{a}{b}, & a<b \\
\frac{b}{a}, & a>b,\end{cases} \\
& I_{00}^{0}(a, b, 0)=\int_{0}^{\infty} J_{0}(a x) J_{0}(b x) d x=\frac{2}{\pi} \frac{1}{a+b} \mathbf{K}(\kappa)=\left\{\begin{array}{cc}
\frac{2}{\pi b} \mathbf{K}\left(\frac{a}{b}\right) & a<b \\
\frac{2}{\pi a} \mathbf{K}\left(\frac{b}{a}\right) & a>b,
\end{array}\right. \\
& I_{01}^{0}(a, b, 0)=\int_{0}^{\infty} J_{0}(a x) J_{1}(b x) d x=\frac{1}{b}[1-H(a-b)]=\frac{1}{b}\left\{\begin{array}{cc}
1 & a<b \\
\frac{1}{2} & a=b \\
0 & a>b,
\end{array}\right. \\
& I_{01}^{-1}(a, b, 0)=\int_{0}^{\infty} \frac{J_{0}(a x) J_{1}(b x)}{x} d x=\frac{1}{\pi b}[(a+b) \mathbf{E}(\kappa)-(a-b) \mathbf{K}(\kappa)] \\
& =\frac{2}{\pi}\left\{\begin{array}{cc}
\mathbf{E}\left(\frac{a}{b}\right) & a<b \\
{\left[\left(\frac{b}{a}-\frac{a}{b}\right) \mathbf{K}\left(\frac{b}{a}\right)+\frac{a}{b} \mathbf{E}\left(\frac{b}{a}\right)\right]} & a>b,
\end{array}\right. \\
& I_{11}^{-1}(a, b, 0)=\int_{0}^{\infty} \frac{J_{1}(a x) J_{1}(b x)}{x} d x=\frac{1}{2} \begin{cases}\frac{a}{b} & a<b \\
\frac{b}{a} & a \geq b,\end{cases} \\
& I_{11}^{0}(a, b, 0)=\int_{0}^{\infty} J_{1}(a x) J_{1}(b x) d x=\frac{a+b}{\pi a b}\left[\frac{a^{2}+b^{2}}{(a+b)^{2}} \mathbf{K}(\kappa)-\mathbf{E}(\kappa)\right] \\
& =\frac{2}{\pi}\left\{\begin{array}{cc}
\frac{1}{a}\left[\mathbf{K}\left(\frac{a}{b}\right)-\mathbf{E}\left(\frac{a}{b}\right)\right] & a<b \\
\infty & a=b \\
\frac{1}{b}\left[\mathbf{K}\left(\frac{b}{a}\right)-\mathbf{E}\left(\frac{b}{a}\right)\right] & a>b,
\end{array}\right.
\end{aligned}
$$




$$
\begin{aligned}
I_{11}^{-2}(a, b, 0) & =\int_{0}^{\infty} \frac{J_{1}(a x) J_{1}(b x)}{x^{2}} d x \\
& =\frac{1}{3 \pi a b}\left[\left(a^{2}+b^{2}\right)(a+b) \mathbf{E}(\kappa)-\left(a^{2}-b^{2}\right)(a-b) \mathbf{K}(\kappa)\right] \\
& =\frac{2}{3 \pi}\left\{\begin{array}{cc}
\frac{1}{a}\left[\left(a^{2}+b^{2}\right) \mathbf{E}\left(\frac{a}{b}\right)-\left(b^{2}-a^{2}\right) \mathbf{K}\left(\frac{a}{b}\right)\right] & a<b \\
2 b & a=b \\
\frac{1}{b}\left[\left(a^{2}+b^{2}\right) \mathbf{E}\left(\frac{b}{a}\right)-\left(a^{2}-b^{2}\right) \mathbf{K}\left(\frac{b}{a}\right)\right] & a \geqslant b .
\end{array}\right.
\end{aligned}
$$

Table 3: Integrals for $a>0, b>0, s>0$, in ENS format. Only the integrals provided by ENS are included in this table. For a more complete list, see the next table.

$$
\begin{aligned}
& \kappa=\frac{2 \sqrt{a b}}{\sqrt{(a+b)^{2}+s^{2}}}, \quad \nu=\frac{4 a b}{(a+b)^{2}} \\
& \mathbf{K}=\mathbf{K}(\kappa), \quad \mathbf{E}=\mathbf{E}(\kappa), \quad \Lambda=\Lambda(\nu, \kappa)=\frac{|a-b|}{a+b} \frac{s}{\sqrt{(a+b)^{2}+s^{2}}} \mathbf{\Pi}(\nu, \kappa) \\
& I_{00}^{1}=\frac{s \kappa^{3}}{4 \pi\left(1-\kappa^{2}\right) a b \sqrt{a b}} \mathbf{E} \\
& I_{10}^{1}=\frac{\kappa}{2 \pi a \sqrt{a b}}\left[\frac{\kappa^{2}\left(a^{2}-b^{2}-s^{2}\right)}{4\left(1-\kappa^{2}\right) a b} \mathbf{E}+\mathbf{K}\right] \\
& I_{11}^{1}=\frac{s \kappa}{2 \pi a b \sqrt{a b}}\left[\left(\frac{1-\frac{1}{2} \kappa^{2}}{1-\kappa^{2}}\right) \mathbf{E}-\mathbf{K}\right] \\
& I_{00}^{0}=\frac{\kappa}{\pi \sqrt{a b}} \mathbf{K}, \\
& I_{10}^{0}=-\frac{1}{\pi a} \frac{\kappa s}{2 \sqrt{a b}} \mathbf{K}-\frac{\operatorname{sgn}(a-b)}{\pi a} \Lambda+\frac{1}{a} H(a-b) \\
& I_{11}^{0}=\frac{2}{\pi \kappa \sqrt{a b}}\left[\left(1-\frac{1}{2} \kappa^{2}\right) \mathbf{K}-\mathbf{E}\right], \\
& I_{10}^{-1}=\frac{1}{\pi a}\left[\frac{2 \sqrt{a b}}{\kappa} \mathbf{E}+\left(a^{2}-b^{2}\right) \frac{\kappa}{2 \sqrt{a b}} \mathbf{K}\right]+\frac{s}{\pi a} \operatorname{sgn}(a-b) \Lambda-\frac{s}{a} H(a-b), \\
& I_{11}^{-1}= \frac{s}{\pi \sqrt{a b}}\left[\frac{1}{\kappa} \mathbf{E}-\frac{\kappa}{2 a b}\left(a^{2}+b^{2}+\frac{1}{2} s^{2}\right) \mathbf{K}\right] \\
&+\frac{1}{4 a b}\left\{a^{2}+b^{2}+\left(a^{2}-b^{2}\right) \operatorname{sgn}(a-b)\left[\frac{2}{\pi} \Lambda-1\right]\right\}
\end{aligned}
$$


Table 4: Integrals for $a>0, b>0, s>0$, in HP format.

$$
\begin{aligned}
& A=\sqrt{(a+b)^{2}+s^{2}}, \quad B=\sqrt{(a-b)^{2}+s^{2}}, \quad L_{1}=\frac{1}{2}(A-B), \quad L_{2}=\frac{1}{2}(A+B), \\
& k=L_{1} / L_{2}, \quad n_{a b}=k \frac{a}{b}, \quad n_{b a}=k \frac{b}{a} \text {, } \\
& \mathbf{K}=\mathbf{K}(k), \quad \mathbf{E}=\mathbf{E}(k), \quad \boldsymbol{\Pi}_{a b}=\boldsymbol{\Pi}\left(n_{a b}, k\right), \quad \boldsymbol{\Pi}_{b a}=\boldsymbol{\Pi}\left(n_{b a}, k\right), \\
& I_{00}^{1}=\frac{2 s}{\pi L_{2}^{3}\left(1-k^{2}\right)}\left[\frac{2}{1-k^{2}} \mathbf{E}-\mathbf{K}\right] \text {, } \\
& I_{10}^{1}=\frac{2}{\pi a L_{2}^{3}\left(1-k^{2}\right)}\left[\left(L_{2}^{2}-a^{2}\right) \mathbf{K}+\frac{a^{2}-b^{2}-s^{2}}{1-k^{2}} \mathbf{E}\right] \text {, } \\
& I_{11}^{1}=\frac{2 s}{\pi a b L_{2}\left(1-k^{2}\right)}\left[\frac{1+k^{2}}{1-k^{2}} \mathbf{E}-\mathbf{K}\right] \text {, } \\
& I_{20}^{1}=\frac{2 s}{\pi L_{2}^{3}\left(1-k^{2}\right)}\left[\mathbf{K}-\frac{2}{1-k^{2}} \mathbf{E}\right]+\frac{4 s}{\pi a^{2} L_{2}}\left(\mathbf{\Pi}_{a b}-\mathbf{K}\right), \quad a<b, \\
& =\frac{2 s}{\pi L_{2}^{3}\left(1-k^{2}\right)}\left[\mathbf{K}-\frac{2}{1-k^{2}} \mathbf{E}\right]+\frac{4 s}{\pi a^{2} L_{2}}\left(\frac{\pi L_{2}}{2 s}-\mathbf{\Pi}_{b a}\right), \quad a>b, \\
& I_{21}^{1}=\frac{2}{\pi a^{2} b L_{2}\left(1-k^{2}\right)}\left\{\left(L_{2}^{2}\left(2-k^{2}\right)-a^{2}\right) \mathbf{K}+\left[\frac{a^{2}\left(s^{2}+a^{2}-b^{2}\right)}{L_{2}^{2}\left(1-k^{2}\right)}-2 L_{2}^{2}\left(1-k^{2}\right)\right] \mathbf{E}\right\} \text {, } \\
& I_{22}^{1}=\frac{2 s L_{2}}{\pi a^{2} b^{2}}\left\{2(\mathbf{E}-\mathbf{K})+\frac{k^{2}}{1-k^{2}}\left[\frac{2}{1-k^{2}} \mathbf{E}-\mathbf{K}\right]\right\} \text {, } \\
& I_{00}^{0}=\frac{2}{\pi L_{2}} \mathbf{K} \\
& I_{10}^{0}=\frac{2 s}{\pi a L_{2}}\left(\boldsymbol{\Pi}_{a b}-\mathbf{K}\right), \quad a<b, \\
& =\frac{2 s}{\pi a L_{2}}\left(\frac{\pi L_{2}}{2 s}-\boldsymbol{\Pi}_{b a}\right), \quad a>b, \\
& I_{11}^{0}=\frac{2}{\pi L_{1}}(\mathbf{K}-\mathbf{E}) \text {, } \\
& I_{20}^{0}=\frac{2 L_{2}}{\pi a^{2}}\left[2(\mathbf{E}-\mathbf{K})+\frac{a^{2}}{L_{2}^{2}} \mathbf{K}+\frac{2 s^{2}}{L_{2}^{2}}\left(\mathbf{K}-\boldsymbol{\Pi}_{a b}\right)\right], \quad a<b, \\
& =\frac{2 L_{2}}{\pi a^{2}}\left[2(\mathbf{E}-\mathbf{K})+\frac{a^{2}}{L_{2}^{2}} \mathbf{K}+\frac{2 s^{2}}{L_{2}^{2}}\left(\boldsymbol{\Pi}_{b a}-\frac{\pi L_{2}}{2 s}\right)\right], \quad a>b, \\
& I_{21}^{0}=\frac{2 s}{\pi a L_{1}}\left[\mathbf{E}-\mathbf{K}+\frac{b^{2}}{L_{2}^{2}}\left(\mathbf{\Pi}_{a b}-\mathbf{K}\right)\right], \quad a<b, \\
& =\frac{2 s}{\pi a L_{1}}\left[\mathbf{E}-\mathbf{K}+\frac{b^{2}}{L_{2}^{2}}\left(\frac{\pi L_{2}}{2 s}-\mathbf{\Pi}_{b a}\right)\right], \quad a>b, \\
& I_{22}^{0}=\frac{2}{3 \pi L_{1}}\left\{\frac{2}{k}(\mathbf{K}-\mathbf{E})+k(\mathbf{K}-2 \mathbf{E})\right\} \text {, }
\end{aligned}
$$




$$
\begin{aligned}
& I_{10}^{-1}=\frac{2 L_{2}}{\pi a}\left[\mathbf{E}-\mathbf{K}+\frac{a^{2}}{L_{2}^{2}} \mathbf{K}+\frac{s^{2}}{L_{2}^{2}}\left(\mathbf{K}-\boldsymbol{\Pi}_{a b}\right)\right], \quad a<b, \\
& =\frac{2 L_{2}}{\pi a}\left[\mathbf{E}-\mathbf{K}+\frac{a^{2}}{L_{2}^{2}} \mathbf{K}+\frac{s^{2}}{L_{2}^{2}}\left(\boldsymbol{\Pi}_{b a}-\frac{\pi L_{2}}{2 s}\right)\right], \quad a>b, \\
& I_{11}^{-1}=\frac{s}{\pi L_{1}}\left[\mathbf{E}-\mathbf{K}+\frac{b^{2}}{L_{2}^{2}}\left(\mathbf{\Pi}_{a b}-\mathbf{K}\right)+\frac{a^{2}}{L_{2}^{2}}\left(\frac{\pi L_{2}}{2 s}-\boldsymbol{\Pi}_{a b}\right)\right], \quad a<b, \\
& =\frac{s}{\pi L_{1}}\left[\mathbf{E}-\mathbf{K}+\frac{a^{2}}{L_{2}^{2}}\left(\boldsymbol{\Pi}_{b a}-\mathbf{K}\right)+\frac{b^{2}}{L_{2}^{2}}\left(\frac{\pi L_{2}}{2 s}-\boldsymbol{\Pi}_{b a}\right)\right], \quad a>b, \\
& I_{20}^{-1}=\frac{s L_{2}}{\pi a^{2}}\left[3(\mathbf{K}-\mathbf{E})-\frac{a^{2}}{L_{2}^{2}} \mathbf{K}+\frac{a^{2}-b^{2}+2 s^{2}}{L_{2}^{2}}\left(\mathbf{\Pi}_{a b}-\mathbf{K}\right)\right], \quad a<b, \\
& =\frac{s L_{2}}{\pi a^{2}}\left[3(\mathbf{K}-\mathbf{E})-\frac{a^{2}}{L_{2}^{2}} \mathbf{K}+\frac{a^{2}-b^{2}+2 s^{2}}{L_{2}^{2}}\left(\frac{\pi L_{2}}{2 s}-\mathbf{\Pi}_{b a}\right)\right], \quad a>b, \\
& I_{21}^{-1}=\frac{2}{3 \pi a L_{1}}\left[\left(2 b^{2}-a^{2}-s^{2}\right)(\mathbf{E}-\mathbf{K})+\frac{a^{2} b^{2}}{L_{2}^{2}} \mathbf{K}+\frac{3 b^{2} s^{2}}{L_{2}^{2}}\left(\mathbf{K}-\mathbf{\Pi}_{a b}\right)\right], \quad a<b, \\
& =\frac{2}{3 \pi a L_{1}}\left[\left(2 b^{2}-a^{2}-s^{2}\right)(\mathbf{E}-\mathbf{K})+\frac{a^{2} b^{2}}{L_{2}^{2}} \mathbf{K}+\frac{3 b^{2} s^{2}}{L_{2}^{2}}\left(\boldsymbol{\Pi}_{b a}-\frac{\pi L_{2}}{2 s}\right)\right], \quad a>b, \\
& I_{22}^{-1}=\frac{s}{6 \pi a b L_{1}}\left[\left(5 a^{2}+5 b^{2}+2 s^{2}\right)(\mathbf{E}-\mathbf{K})+\frac{a^{2} b^{2}}{L_{2}^{2}} \mathbf{K}+\frac{3 b^{4}}{L_{2}^{2}}\left(\mathbf{\Pi}_{a b}-\mathbf{K}\right)\right. \\
& \left.+\frac{3 a^{4}}{L_{2}^{2}}\left(\frac{\pi L_{2}}{2 s}-\Pi_{a b}\right)\right], \quad a<b, \\
& =\frac{s}{6 \pi a b L_{1}}\left[\left(5 a^{2}+5 b^{2}+2 s^{2}\right)(\mathbf{E}-\mathbf{K})+\frac{a^{2} b^{2}}{L_{2}^{2}} \mathbf{K}+\frac{3 a^{4}}{L_{2}^{2}}\left(\mathbf{\Pi}_{b a}-\mathbf{K}\right)\right. \\
& \left.+\frac{3 b^{4}}{L_{2}^{2}}\left(\frac{\pi L_{2}}{2 s}-\Pi_{b a}\right)\right], \quad a>b, \\
& I_{11}^{-2}=\frac{1}{3 \pi L_{1}}\left[\left(2 a^{2}+2 b^{2}-s^{2}\right)(\mathbf{E}-\mathbf{K})+\frac{4 a^{2} b^{2}}{L_{2}^{2}} \mathbf{K}+\frac{3 b^{2} s^{2}}{L_{2}^{2}}\left(\mathbf{K}-\boldsymbol{\Pi}_{a b}\right)\right. \\
& \left.+\frac{3 a^{2} s^{2}}{L_{2}^{2}}\left(\Pi_{a b}-\frac{\pi L_{2}}{2 s}\right)\right], \quad a<b, \\
& =\frac{1}{3 \pi L_{1}}\left[\left(2 a^{2}+2 b^{2}-s^{2}\right)(\mathbf{E}-\mathbf{K})+\frac{4 a^{2} b^{2}}{L_{2}^{2}} \mathbf{K}+\frac{3 a^{2} s^{2}}{L_{2}^{2}}\left(\mathbf{K}-\boldsymbol{\Pi}_{b a}\right)\right. \\
& \left.+\frac{3 b^{2} s^{2}}{L_{2}^{2}}\left(\Pi_{b a}-\frac{\pi L_{2}}{2 s}\right)\right], \quad a>b
\end{aligned}
$$




$$
\begin{aligned}
I_{20}^{-2}= & \frac{L_{2}}{9 \pi a^{2}}\left[\left(8 a^{2}-4 b^{2}+11 s^{2}\right)(\mathbf{E}-\mathbf{K})+\frac{a^{2}\left(6 a^{2}-2 b^{2}+3 s^{2}\right)}{L_{2}^{2}} \mathbf{K}\right. \\
& \left.+\frac{3 s^{2}\left(3 a^{2}-3 b^{2}+2 s^{2}\right)}{L_{2}^{2}}\left(\mathbf{K}-\mathbf{\Pi}_{a b}\right)\right], \quad a<b, \\
= & \frac{L_{2}}{9 \pi a^{2}}\left[\left(8 a^{2}-4 b^{2}+11 s^{2}\right)(\mathbf{E}-\mathbf{K})+\frac{a^{2}\left(6 a^{2}-2 b^{2}+3 s^{2}\right)}{L_{2}^{2}} \mathbf{K}\right. \\
& \left.+\frac{3 s^{2}\left(3 a^{2}-3 b^{2}+2 s^{2}\right)}{L_{2}^{2}}\left(\boldsymbol{\Pi}_{b a}-\frac{\pi L_{2}}{2 s}\right)\right], \quad a>b, \\
I_{21}^{-2}= & \frac{s}{12 \pi a L_{1}}\left[\left(5 a^{2}-13 b^{2}+2 s^{2}\right)(\mathbf{E}-\mathbf{K})-\frac{a^{2}\left(3 a^{2}+5 b^{2}\right)}{L_{2}^{2}} \mathbf{K}\right. \\
& \left.+\frac{3\left(a^{2}-b^{2}\right)^{2}-12 b^{2} s^{2}}{L_{2}^{2}}\left(\mathbf{K}-\mathbf{\Pi}_{a b}\right)+\frac{3 \pi L_{2} a^{4}}{2 s L_{2}^{2}}\right], \quad a<b, \\
= & \frac{s}{12 \pi a L_{1}}\left[\left(5 a^{2}-13 b^{2}+2 s^{2}\right)(\mathbf{E}-\mathbf{K})-\frac{a^{2}\left(3 a^{2}+5 b^{2}\right)}{L_{2}^{2}} \mathbf{K}\right. \\
& \left.+\frac{3\left(a^{2}-b^{2}\right)^{2}-12 b^{2} s^{2}}{L_{2}^{2}}\left(\mathbf{\Pi}_{b a}-\frac{\pi L_{2}}{2 s}\right)+\frac{3 \pi L_{2} a^{4}}{2 s L_{2}^{2}}\right], \quad a>b, \\
I_{22}^{-2}= & \frac{1}{30 \pi a b L_{1}}\left\{\left[8\left(a^{2}-b^{2}\right)^{2}+8 a^{2} b^{2}-s^{2}\left(9 a^{2}+9 b^{2}+2 s^{2}\right)\right](\mathbf{E}-\mathbf{K})\right. \\
& \left.+a^{2} b^{2} \frac{4\left(a^{2}+b^{2}\right)-s^{2}}{L_{2}^{2}} \mathbf{K}+15 \frac{b^{4} s^{2}}{L_{2}^{2}}\left(\mathbf{K}-\mathbf{\Pi}_{a b}\right)+15 \frac{a^{4} s^{2}}{L_{2}^{2}}\left(\boldsymbol{\Pi}_{a b}-\frac{\pi L_{2}}{2 s}\right)\right\}, a<b, \\
= & \frac{1}{30 \pi a b L_{1}}\left\{\left[8\left(a^{2}-b^{2}\right)^{2}+8 a^{2} b^{2}-s^{2}\left(9 a^{2}+9 b^{2}+2 s^{2}\right)\right](\mathbf{E}-\mathbf{K})\right. \\
& \left.+a^{2} b^{2} \frac{4\left(a^{2}+b^{2}\right)-s^{2}}{L_{2}^{2}} \mathbf{K}+15 \frac{a^{4} s^{2}}{L_{2}^{2}}\left(\mathbf{K}-\mathbf{\Pi}_{b a}\right)+15 \frac{b^{4} s^{2}}{L_{2}^{2}}\left(\Pi_{b a}-\frac{\pi L_{2}}{2 s}\right)\right\}, a>b .
\end{aligned}
$$


Appendix A. Proof of transformation formula. Eason, Noble and Sneddon define the scaled Heuman function

$$
\begin{aligned}
\Lambda(\psi, \kappa) & =\frac{\sqrt{(1-\nu)\left(\nu-\kappa^{2}\right)}}{\sqrt{\nu}} \boldsymbol{\Pi}(\nu, \kappa)=\frac{|a-b|}{(a+b)} \frac{s}{\sqrt{(a+b)^{2}+s^{2}}} \boldsymbol{\Pi}(\nu, \kappa) \\
& =[\mathbf{E}(\kappa)-\mathbf{K}(\kappa)] F\left(\psi, \kappa^{\prime}\right)+\mathbf{K}(\kappa) E\left(\psi, \kappa^{\prime}\right),
\end{aligned}
$$

where

$$
\sin \psi=\frac{1}{\kappa^{\prime}} \sqrt{\frac{\nu-\kappa^{2}}{\nu}}=\frac{s}{B}, \quad \cos \psi=\frac{\kappa}{\kappa^{\prime}} \sqrt{\frac{1-\nu}{\nu}}=\frac{|a-b|}{B}, \quad \tan \psi=\frac{s}{|a-b|} .
$$

Thus, we need expressions to move from one set of parameters to the other. From Gradshteyn and Ryzhik, page 907, formulas 8.121-3, 8.121-4, and page 908, formulas $8.125,8.126$, the Landen-Gauss transformations which are relevant to this proof are:

$$
\begin{aligned}
& \begin{array}{ll|l}
\kappa=\frac{2 \sqrt{k}}{1+k}, & \mathbf{K}(\kappa)=(1+k) \mathbf{K}(k) & \mathbf{E}(\kappa)=\frac{2}{1+k} \mathbf{E}(k)-(1-k) \mathbf{K}(k) \\
\hline
\end{array} \\
& \kappa^{\prime}=\frac{1-k}{1+k}, \quad F\left(\psi, \kappa^{\prime}\right)=(1+k) F\left(\varphi, k^{\prime}\right) \\
& E\left(\psi, \kappa^{\prime}\right)=\frac{2}{1+k}\left[E\left(\varphi, k^{\prime}\right)+k F\left(\varphi, k^{\prime}\right)\right]-\frac{1-k}{1+k} \sin \psi
\end{aligned}
$$

where

$$
\tan (\psi-\varphi)=k \tan \varphi
$$

Substituting these into the expansion of the scaled Heuman function above, we obtain

$$
\begin{aligned}
\Lambda(\psi, \kappa)= & {[\mathbf{E}(\kappa)-\mathbf{K}(\kappa)] F\left(\psi, \kappa^{\prime}\right)+\mathbf{K}(\kappa) E\left(\psi, \kappa^{\prime}\right) } \\
= & {\left[\frac{2}{1+k} \mathbf{E}(k)-(1-k) \mathbf{K}(k)-(1+k) \mathbf{K}(k)\right](1+k) F\left(\varphi, k^{\prime}\right) } \\
& +(1+k) \mathbf{K}(k)\left\{\frac{2}{1+k}\left[E\left(\varphi, k^{\prime}\right)+k F\left(\varphi, k^{\prime}\right)\right]-\frac{1-k}{1+k} \sin \psi\right\} \\
= & 2\left\{[\mathbf{E}(k)-\mathbf{K}(k)] F\left(\varphi, k^{\prime}\right)+\mathbf{K}(k) E\left(\varphi, k^{\prime}\right)\right\}-(1-k) \sin \psi \mathbf{K}(k) .
\end{aligned}
$$

But

so

$$
(1-k) \sin \psi=\left(1-\frac{L_{1}}{L_{2}}\right) \frac{s}{L_{2}-L_{1}}=\frac{s}{L_{2}}
$$

$$
\Lambda(\psi, \kappa)=2 \Lambda(\varphi, k)-\frac{s}{L_{2}} \mathbf{K}(k)
$$

Also, expanding the expression for $\tan (\psi-\varphi)$, we obtain

$$
k \tan \psi \tan ^{2} \varphi+(1+k) \tan \varphi-\tan \psi=0,
$$

which is a quadratic equation in $\tan \varphi$. Its solution is

$$
\begin{aligned}
\tan \varphi & =-\frac{1+k}{2 k \tan \psi}\left\{1 \mp \sqrt{1+\frac{4 k}{(1+k)^{2}} \tan ^{2} \psi}\right\} \\
& =-\frac{1}{\left(1-\kappa^{\prime}\right) \tan \psi}\left\{1 \mp \sqrt{1+\kappa^{2} \tan ^{2} \psi}\right\} .
\end{aligned}
$$


But

$$
\kappa \tan \psi=\sqrt{1-\kappa^{\prime 2}} \frac{s}{|a-b|}
$$

and

$$
\begin{aligned}
\sqrt{1+\kappa^{2} \tan ^{2} \psi} & =\sqrt{1+\frac{4 a b s^{2}}{\left[(a+b)^{2}+s^{2}\right](a-b)^{2}}} \\
& =\sqrt{\frac{(a+b)^{2}(a-b)^{2}+s^{2}(a-b)^{2}+4 a b s^{2}}{\left[(a+b)^{2}+s^{2}\right](a-b)^{2}}} \\
& =\frac{a+b}{|a-b|} \frac{\sqrt{(a-b)^{2}+s^{2}}}{\sqrt{(a+b)^{2}+s^{2}}}=\frac{a+b}{|a-b|} \kappa^{\prime} .
\end{aligned}
$$

Hence, taking into account that $k=\left(1-\kappa^{\prime}\right) /\left(1+\kappa^{\prime}\right)$, then

$$
\begin{aligned}
\tan \varphi & =-\frac{|a-b|}{\left(1-\kappa^{\prime}\right) s}\left\{1 \pm \frac{a+b}{|a-b|} \kappa^{\prime}\right\}=\frac{1}{\left(1-\kappa^{\prime}\right) s}\left\{-|a-b| \mp(a+b) \kappa^{\prime}\right\} \\
& =\frac{\operatorname{sgn}(a-b)}{k s}\left\{\begin{array}{l}
b k-a \\
b-a k .
\end{array}\right.
\end{aligned}
$$

A) Let's consider the first of the two solutions above. In this case,

$$
\tan \varphi=\frac{b k-a}{k s} \operatorname{sgn}(a-b)=\frac{s^{2} k b}{\left(L_{1}^{2}-b^{2}\right) k s} \operatorname{sgn}(a-b)=\frac{s}{b\left(n_{a b}-1\right)} \operatorname{sgn}(a-b),
$$

so

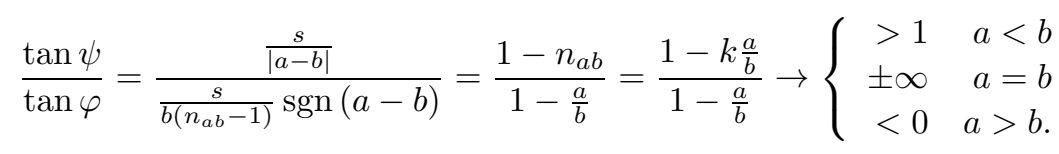

Case 1: $a<b$

Here $\tan \psi>\tan \varphi$, i.e., $\psi>\varphi$ and $\psi-\varphi>0$. Also, $k \tan \varphi>0$, in which case the angle transformation formula $\tan (\psi-\varphi)=k \tan \varphi$ is satisfied with positive angles in the range $\left[0, \frac{1}{2} \pi\right]$. Thus, in this case,

$$
\begin{gathered}
\tan \varphi=\frac{1}{k} \sqrt{\frac{n_{a b}-k^{2}}{1-n_{a b}}}=\frac{s}{b\left(1-n_{a b}\right)}, \\
\sin \varphi=\frac{1}{k^{\prime}} \sqrt{\frac{n_{a b}-k^{2}}{n_{a b}}}=\frac{s}{k^{\prime} L_{2} \sqrt{1-n_{a b}}}, \\
\cos \varphi=\frac{k}{k^{\prime}} \sqrt{\frac{1-n_{a b}}{n_{a b}}}=\frac{b \sqrt{1-n_{a b}}}{k^{\prime} L_{2}} .
\end{gathered}
$$

Also

$$
\Lambda(\varphi, k) \equiv \Lambda_{a b}=\sqrt{\frac{\left(1-n_{a b}\right)\left(n_{a b}-k^{2}\right)}{n_{a b}}} \Pi\left(n_{a b}, k\right)=\frac{s}{L_{2}} \mathbf{\Pi}_{a b},
$$


so

$$
\Lambda(\psi, \kappa)=\frac{s}{L_{2}}\left[2 \mathbf{\Pi}_{a b}-\mathbf{K}\right] \quad a<b .
$$

Case 2: $a>b$

Now $\tan \varphi<0$, i.e. $\varphi<0$, which we reject.

B) Consider next the second solution for $\tan \varphi$ :

$$
\tan \varphi=\frac{b-a k}{k s} \operatorname{sgn}(a-b)=\frac{s a}{a^{2}-L_{1}^{2}} \operatorname{sgn}(a-b)=\frac{s}{a\left(1-n_{b a}\right)} \operatorname{sgn}(a-b),
$$

SO

$$
\frac{\tan \psi}{\tan \varphi}=\frac{\frac{s}{|a-b|}}{\frac{s}{a\left(1-n_{b a}\right)} \operatorname{sgn}(a-b)}=\frac{1-n_{b a}}{1-\frac{b}{a}}=\frac{1-k \frac{b}{a}}{1-\frac{b}{a}} \rightarrow\left\{\begin{array}{cc}
<0 & a<b \\
\pm \infty & a=b \\
>1 & a>b
\end{array}\right.
$$

We see that when $a<b$ we obtain a negative solution $\varphi<0$, so we reject it as well. On the other hand, for $a>b$ this leads us to

$$
\begin{gathered}
\tan \varphi=\frac{1}{k} \sqrt{\frac{n_{b a}-k^{2}}{1-n_{b a}}}=\frac{s}{a\left(1-n_{b a}\right)}, \\
\sin \varphi=\frac{1}{k^{\prime}} \sqrt{\frac{n_{b a}-k^{2}}{n_{b a}}}=\frac{s}{k^{\prime} L_{2} \sqrt{1-n_{b a}}}, \\
\cos \varphi=\frac{k}{k^{\prime}} \sqrt{\frac{1-n_{b a}}{n_{b a}}}=\frac{a \sqrt{1-n_{b a}}}{k^{\prime} L_{2}},
\end{gathered}
$$

and

So

$$
\Lambda(\varphi, k) \equiv \Lambda_{b a}=\sqrt{\frac{\left(1-n_{b a}\right)\left(n_{b a}-k^{2}\right)}{n_{b a}}} \Pi\left(n_{b a}, k\right)=\frac{s}{L_{2}} \boldsymbol{\Pi}_{b a}
$$

$$
\Lambda(\psi, \kappa)=\frac{s}{L_{2}}\left[2 \mathbf{\Pi}_{b a}-\mathbf{K}\right] \quad a>b .
$$

An additional useful formula is derived next. From section 3, the characteristics of $\boldsymbol{\Pi}_{a b}, \boldsymbol{\Pi}_{b a}$ are $n_{a b}=k \frac{a}{b}>k^{2}$ and $n_{b a}=k \frac{b}{a}>k^{2}$, which together satisfy the relationship $n_{a b} n_{b a}=k^{2}$. Hence, the special addition formula 117.02 on page 13 in Byrd and Friedman applies to these functions:

$$
\begin{aligned}
\boldsymbol{\Pi}\left(k \frac{a}{b}, k\right)+\boldsymbol{\Pi}\left(k \frac{b}{a}, k\right) & =\mathbf{K}(k)+\frac{\pi}{2} \sqrt{\frac{k \frac{a}{b}}{\left(1-k \frac{a}{b}\right)\left(k \frac{a}{b}-k^{2}\right)}} \\
& =\mathbf{K}(k)+\frac{\pi}{2} \sqrt{\frac{1}{k^{2}-\left(\frac{a}{b}+\frac{b}{a}\right) k+1}} .
\end{aligned}
$$

Also

$$
k\left(\frac{a}{b}+\frac{b}{a}\right)=\frac{L_{1}}{L_{2}} \frac{a^{2}+b^{2}}{a b}=\frac{a^{2}+b^{2}}{L_{2}^{2}}=\frac{L_{1}^{2}+L_{2}^{2}-s^{2}}{L_{2}^{2}} \quad \text { and } \quad k^{2}=\frac{L_{1}^{2}}{L_{2}^{2}},
$$


$\mathrm{SO}$

$$
\sqrt{1-k\left(\frac{a}{b}+\frac{b}{a}\right)+k^{2}}=\sqrt{1-\frac{L_{1}^{2}+L_{2}^{2}-s^{2}}{L_{2}^{2}}+\frac{L_{1}^{2}}{L_{2}^{2}}}=\sqrt{\frac{s^{2}}{L_{2}^{2}}}=\frac{s}{L_{2}} .
$$

Hence

$$
\mathbf{\Pi}_{a b}+\boldsymbol{\Pi}_{b a}=\mathbf{K}(k)+\frac{\pi L_{2}}{2 s}
$$

Appendix B. Derivatives and continuity of Lambda function. It can be shown that

$$
\begin{gathered}
\frac{\partial \boldsymbol{\Pi}\left(n_{a b}, k\right)}{\partial a}=\frac{\partial \boldsymbol{\Pi}_{a b}}{\partial n_{a b}} \frac{\partial n_{a b}}{\partial a}+\frac{\partial \boldsymbol{\Pi}_{a b}}{\partial k} \frac{\partial k}{\partial a}=\frac{\partial \boldsymbol{\Pi}_{a b}}{\partial n_{a b}}\left(\frac{k}{b}+\frac{a}{b} \frac{\partial k}{\partial a}\right)+\frac{\partial \boldsymbol{\Pi}_{a b}}{\partial k} \frac{\partial k}{\partial a} \\
=\frac{b}{2 k a(k a-b)}\left(k+a \frac{\partial k}{\partial a}\right)\left(\mathbf{K}(k)+\frac{a}{k b-a} \mathbf{E}(k)-b k \frac{1-\left(\frac{a}{b}\right)^{2}}{k b-a} \mathbf{\Pi}_{a b}\right) \\
\quad+\frac{b}{a-k b}\left(\boldsymbol{\Pi}_{a b}-\frac{\mathbf{E}(k)}{1-k^{2}}\right) \frac{\partial k}{\partial a} \\
\frac{\partial \boldsymbol{\Pi}_{b a}}{\partial a}=\frac{\partial \boldsymbol{\Pi}_{b a}}{\partial n_{b a}} \frac{\partial n_{b a}}{\partial a}+\frac{\partial \boldsymbol{\Pi}_{b a}}{\partial k} \frac{\partial k}{\partial a}=\frac{b}{a^{2}}\left(a \frac{\partial k}{\partial a}-k\right) \frac{\partial \boldsymbol{\Pi}_{b a}}{\partial n_{b a}}+\frac{\partial \boldsymbol{\Pi}_{b a}}{\partial k} \frac{\partial k}{\partial a} \\
=\frac{1}{2 k(k b-a)}\left(a \frac{\partial k}{\partial a}-k\right)\left(\mathbf{K}(k)+\frac{b}{a k-b} \mathbf{E}(k)-a k \frac{1-\left(\frac{b}{a}\right)^{2}}{a k-b} \mathbf{\Pi}_{b a}\right) \\
+\frac{a}{b-a k}\left(\boldsymbol{\Pi}_{b a}-\frac{\mathbf{E}(k)}{1-k^{2}}\right) \frac{\partial k}{\partial a} .
\end{gathered}
$$

When $a=b$, then

$$
\begin{aligned}
\left.\frac{\partial \boldsymbol{\Pi}_{a b}}{\partial a}\right|_{a=b}= & \frac{1}{2 k b(1-k)}\left(k+\left.b \frac{\partial k}{\partial a}\right|_{a=b}\right)\left(\frac{1}{1-k} \mathbf{E}(k)-\mathbf{K}(k)\right) \\
& +\left.\frac{1}{1-k} \frac{\partial k}{\partial a}\right|_{a=b}\left(\boldsymbol{\Pi}_{a b}-\frac{\mathbf{E}(k)}{1-k^{2}}\right), \\
\left.\frac{\partial \boldsymbol{\Pi}_{b a}}{\partial a}\right|_{a=b}= & \frac{1}{2 k b(1-k)}\left(\left.b \frac{\partial k}{\partial a}\right|_{a=b}-k\right)\left(\frac{1}{1-k} \mathbf{E}(k)-\mathbf{K}(k)\right) \\
& +\left.\frac{1}{1-k} \frac{\partial k}{\partial a}\right|_{a=b}\left(\boldsymbol{\Pi}_{b a}-\frac{\mathbf{E}(k)}{1-k^{2}}\right),
\end{aligned}
$$

and from their difference,

$$
\left.\left(\frac{\partial \boldsymbol{\Pi}_{a b}}{\partial a}-\frac{\partial \boldsymbol{\Pi}_{b a}}{\partial a}\right)\right|_{a=b}=\frac{1}{b(1-k)}\left(\frac{1}{1-k} \mathbf{E}(k)-\mathbf{K}(k)\right)+\left.\left.\frac{1}{1-k} \frac{\partial k}{\partial a}\right|_{a=b}\left(\boldsymbol{\Pi}_{a b}-\boldsymbol{\Pi}_{b a}\right)\right|_{a=b} .
$$

Since $\left.\left(\boldsymbol{\Pi}_{a b}-\boldsymbol{\Pi}_{b a}\right)\right|_{a=b}=0$, then

$$
\left.\left(\frac{\partial \mathbf{\Pi}_{a b}}{\partial a}-\frac{\partial \mathbf{\Pi}_{b a}}{\partial a}\right)\right|_{a=b}=\left.\frac{1}{b(1-k)}\left(\frac{1}{1-k} \mathbf{E}(k)-\mathbf{K}(k)\right)\right|_{a=b} \neq 0
$$

which shows that $\Pi_{a b}, \Pi_{b a}$ do not continue one into the other at $a=b$, but have distinct slopes at this transitional value. 
Consider next the ENS Lambda function:

$$
\Lambda(\nu, \kappa)=\frac{\sqrt{(1-\nu)\left(\nu-\kappa^{2}\right)}}{\sqrt{\nu}} \boldsymbol{\Pi}(\nu, \kappa)=\frac{\pi}{2} \Lambda_{0}(\psi \backslash \vartheta) .
$$

When $a=b$, then $\nu=1$ with $\kappa<1$ if $s>0$, in which case $\Pi(1, \kappa)=\infty$. However, $\Lambda(\nu, \kappa)$ remains finite because of the factor $\sqrt{(1-\nu)} \rightarrow 0$. The proof of this relies on the relationship between the ENS Lambda function and Heuman's function. Indeed, when $\nu=1 \rightarrow \cos ^{2} \psi=0 \rightarrow \psi=\frac{1}{2} \pi$, we have

$$
\Lambda(1, \kappa)=\frac{1}{2} \pi \Lambda_{0}\left(\frac{1}{2} \pi \backslash \vartheta\right)=\frac{\pi}{2} \times 1=\frac{\pi}{2}, \quad \text { for any } \kappa<1 \quad\left(\vartheta<\frac{1}{2} \pi\right), \quad a=b,
$$

so the Lambda function remains finite at $a=b$. On the other hand, in Appendix A we found the equivalence

$$
\Lambda(\nu, \kappa)=\left\{\begin{array}{l}
\Lambda_{a b} \\
\Lambda_{b a}
\end{array}=\frac{s}{L_{2}} \begin{cases}2 \boldsymbol{\Pi}_{a b}-\mathbf{K}(k), & a<b \\
2 \boldsymbol{\Pi}_{b a}-\mathbf{K}(k), & a>b,\end{cases}\right.
$$

which we have verified to be true by numerical testing. Since $\left.\boldsymbol{\Pi}_{a b}\right|_{a=b}=\left.\Pi_{b a}\right|_{a=b}$, it follows that $\left.\Lambda_{a b}\right|_{a=b}=\left.\Lambda_{b a}\right|_{a=b}=\Lambda(1, \kappa)=\frac{1}{2} \pi$, so the Lambda function itself is continuous at $a=b$. However, as we have already found out in (B.4),

$$
\left.\frac{\partial \boldsymbol{\Pi}_{a b}}{\partial a}\right|_{a=b} \neq\left.\frac{\partial \boldsymbol{\Pi}_{b a}}{\partial a}\right|_{a=b} .
$$

Hence, $\Lambda$ exhibits a discontinuity of slope when $a=b$. On the other hand, the modified function

$$
\operatorname{sgn}(a-b) \Lambda(\nu, \kappa)=\frac{a-b}{a+b} \frac{s}{L_{2}(1+k)} \Pi(\nu, \kappa)
$$

jumps from -1 through 0 to +1 in the neighborhood $a=[b-\varepsilon, b, b+\varepsilon]$. Hence, the equivalence

$$
\operatorname{sgn}(a-b) \Lambda(\nu, \kappa)=\frac{s}{L_{2}} \begin{cases}\mathbf{K}(k)-2 \boldsymbol{\Pi}_{a b}, & a<b \\ 2 \boldsymbol{\Pi}_{b a}-\mathbf{K}(k), & a>b\end{cases}
$$

is intrinsically discontinuous, so HP's use of the upper expression in the domain $a>b$ led them necessarily to an erroneous branch. Nonetheless, despite the intrinsic discontinuity of the Lambda function, many of the integrals and particularly those that affect HP's elasticity problem are still continuous up to first order; i.e., strains and stresses are continuous. For example, in (5.1b), the discontinuous fragment is

$$
f(a, b, s)=\operatorname{sgn}(a-b)\left[\frac{2}{\pi} \Lambda(\nu, \kappa)-1\right] .
$$

Since as we have just seen that $\frac{2}{\pi} \Lambda(1, \kappa)=1$, then $f(a, a, s)=\operatorname{sgn}(a-b) \times 0=0$, both when approaching from the left or from the right, so the primitive fragment is continuous. Also, the first derivative will contain a term of the form

$$
\left[\frac{2}{\pi} \Lambda(\nu, \kappa)-1\right] \delta(a-b)
$$

which despite the Dirac-delta factor is also zero at the transition, so again the first derivative of the fragment is continuous. Higher derivatives, however, will be discontinuous.

The previous results led to an interesting corollary. Since at $a=b, \Lambda_{a a}=\frac{1}{2} \pi$ and $\Pi_{a a}=\Pi(k, k)$, then

$$
2 \boldsymbol{\Pi}(k, k)-\mathbf{K}(k)=\pi \frac{L_{2}}{2 s},
$$


where

$$
L_{1}=b\left(\sqrt{1+\left(\frac{s}{2 b}\right)^{2}}-\frac{s}{2 b}\right), \quad L_{2}=b\left(\sqrt{1+\left(\frac{s}{2 b}\right)^{2}}+\frac{s}{2 b}\right)
$$

and

$$
\begin{gathered}
\frac{2 s}{L_{2}}=2 \frac{s}{b} \frac{b}{L_{2}}=2 \frac{L_{2}-L_{1}}{L_{2}}=2\left(1-\frac{L_{1}}{L_{2}}\right)=2(1-k), \\
k=\frac{L_{1}}{L_{2}}=\frac{L_{1}^{2}}{b^{2}}=\frac{b^{2}}{L_{2}^{2}},
\end{gathered}
$$

so

$$
2 \boldsymbol{\Pi}(k, k)-\mathbf{K}(k)=\frac{\pi}{2(1-k)}
$$

or

$$
(1-k)[2 \boldsymbol{\Pi}(k, k)-\mathbf{K}(k)]=\frac{\pi}{2}
$$

which is valid for any $0<k<1$. Of course, this is nothing but an alternative representation of Heuman's lambda function satisfying the limiting condition

$$
\left.\Lambda_{0}(\nu, \kappa)\right|_{\nu=1}=\Lambda_{0}\left(\frac{1}{2} \pi \backslash \vartheta\right)=1 .
$$

Appendix C. Numerical integration. To avoid mistakes, all of the integration formulas given earlier have been verified by direct numerical integration. To this purpose, the improper integrals are divided into two integration ranges $\left[0, x_{0}\right]$ and $\left[x_{0}, \infty\right]$, where $x_{0}$ defines the start of the tail. The body is integrated by an appropriate numerical quadrature which accounts for the rate of change of the integrands, while the tail is obtained in closed-form using asymptotic expansions as follows:

$$
\begin{gathered}
J_{m}(a x) \approx \sqrt{\frac{2}{\pi a x}} \cos \left[a x-\frac{1}{4} \pi(1+2 m)\right], \\
J_{n}(b x) \approx \sqrt{\frac{2}{\pi b x}} \cos \left[b x-\frac{1}{4} \pi(1+2 n)\right], \\
J_{m}(a x) J_{n}(b x) \approx \frac{2}{\pi} \frac{1}{x \sqrt{a b}} \cos \left[a x-\frac{1}{4} \pi(1+2 m)\right] \cos \left[b x-\frac{1}{4} \pi(1+2 n)\right] .
\end{gathered}
$$

But

$$
\cos \varphi \cos \psi=\frac{1}{2}\{\cos (\varphi-\psi)+\cos (\varphi+\psi)\}
$$

so

$$
\begin{aligned}
\cos [ & \left.a x-\frac{1}{4} \pi(1+2 m)\right] \cos \left[b x-\frac{1}{4} \pi(1+2 n)\right] \\
= & \frac{1}{2}\left\{\cos \left[(a-b) x-\frac{1}{2} \pi(m-n)\right]+\cos \left[(a+b) x-\frac{1}{2} \pi(m+n+1)\right]\right\} \\
= & \frac{1}{2}\left\{\cos (a-b) x \cos \frac{1}{2} \pi(m-n)+\sin (a-b) x \sin \frac{1}{2} \pi(m-n)\right. \\
& \left.+\cos (a+b) x \cos \frac{1}{2} \pi(m+n+1)+\sin (a+b) x \sin \frac{1}{2} \pi(m+n+1)\right\} .
\end{aligned}
$$


Also

$$
\begin{aligned}
\cos \frac{1}{2} \pi(m-n) & =\left\{\begin{array}{cc}
(-1)^{\frac{1}{2}(m-n)} & m-n \text { is even } \\
0 & m-n \text { is odd, }
\end{array}\right. \\
\cos \frac{1}{2} \pi(m+n+1) & =\left\{\begin{array}{cc}
0 & m+n \text { is even } \\
(-1)^{\frac{1}{2}(m+n+1)} & m+n \text { is odd, }
\end{array}\right. \\
\sin \frac{1}{2} \pi(m-n) & =\left\{\begin{array}{cc}
0 & m-n \text { is even } \\
(-1)^{\frac{1}{2}(m-n-1)} & m-n \text { is odd },
\end{array}\right. \\
\sin \frac{1}{2} \pi(m+n+1) & =\left\{\begin{array}{cc}
(-1)^{\frac{1}{2}(m+n)} & m+n \text { is even } \\
0 & m+n \text { is odd. }
\end{array}\right.
\end{aligned}
$$

Then again, $m \pm n$ is even if both $m, n$ are even, or both are odd, and it is odd if one is even and the other one is odd. Hence,

$$
\begin{array}{r}
J_{m}(a x) J_{n}(b x) \approx \frac{1}{\pi x \sqrt{a b}}\left[(-1)^{\frac{1}{2}(m-n)} \cos (a-b) x+(-1)^{\frac{1}{2}(m+n)} \sin (a+b) x\right] \\
\text { even } \quad m \pm n, \\
J_{m}(a x) J_{n}(b x) \approx \frac{1}{\pi x \sqrt{a b}}\left[(-1)^{\frac{1}{2}(m-n-1)} \sin (a-b) x+(-1)^{\frac{1}{2}(m+n+1)} \cos (a+b) x\right] \\
\text { odd } m \pm n .
\end{array}
$$

Observe that $(-1)^{\frac{1}{2}(m+n)}=(-1)^{\frac{1}{2}(m-n)}(-1)^{n} \neq(-1)^{\frac{1}{2}(m-n)}$.

We now define the following exponential integrals:

$$
\begin{aligned}
& C_{0}(A, s)=\int_{x_{0}}^{\infty} e^{-s x} \cos A x d x=\frac{s \cos A x_{0}-A \sin A x_{0}}{A^{2}+s^{2}} e^{-s x_{0}}, \\
& S_{0}(A, s)=\int_{x_{0}}^{\infty} e^{-s x} \cos A x d x=\frac{A \cos A x_{0}+s \sin A x_{0}}{A^{2}+s^{2}} e^{-s x_{0}}, \\
& C_{-1}(A, s)=\int_{x_{0}}^{\infty} e^{-s x} \frac{\cos A x}{x} d x=\operatorname{Re}\left\{E_{1}\left[x_{0}(s+i A)\right]\right\}, \\
& S_{-1}(A, s)=\int_{x_{0}}^{\infty} e^{-s x} \frac{\sin A x}{x} d x=-\operatorname{Im}\left\{E_{1}\left[x_{0}(s+i A)\right]\right\} \\
& C_{-2}(A, s)=\int_{x_{0}}^{\infty} e^{-s x} \frac{\cos A x}{x^{2}} d x=\frac{e^{-s x_{0}} \cos A x_{0}-\operatorname{Re}\left\{x_{0}(s+i A) E_{1}\left[x_{0}(s+i A)\right]\right\}}{x_{0}}, \\
& S_{-2}(A, s)=\int_{x_{0}}^{\infty} e^{-s x} \frac{\sin A x}{x^{2}} d x=\frac{e^{-s x_{0}} \sin A x_{0}+\operatorname{Im}\left\{x_{0}(s+i A) E_{1}\left[x_{0}(s+i A)\right]\right\}}{x_{0}},
\end{aligned}
$$

where $E_{1}(z)=$ exponential integral, which can readily be evaluated using Matlab. We can now express the tail $T_{m n}^{\lambda}(a, b, s)$ of the integrals as: 
Even $m \pm n$ :

$$
\begin{aligned}
T_{m n}^{\lambda}(a, b, s) & =\int_{x_{0}}^{\infty} e^{-s x} x^{\lambda} J_{m}(a x) J_{n}(b x) d x \\
& =\frac{1}{\pi \sqrt{a b}}\left[(-1)^{\frac{1}{2}(m-n)} C_{\lambda-1}(a-b, s)+(-1)^{\frac{1}{2}(m+n)} S_{\lambda-1}(a+b, s)\right]
\end{aligned}
$$

Odd $m \pm n$ :

$$
\begin{aligned}
T_{m n}^{\lambda}(a, b, s) & =\int_{x_{0}}^{\infty} e^{-s x} x^{\lambda} J_{m}(a x) J_{n}(b x) d x \\
& =\frac{1}{\pi \sqrt{a b}}\left[(-1)^{\frac{1}{2}(m-n-1)} S_{\lambda-1}(a-b, s)+(-1)^{\frac{1}{2}(m+n+1)} C_{\lambda-1}(a+b, s)\right]
\end{aligned}
$$

\section{REFERENCES}

[1] M. Abramovitz and I. Stegun. Handbook of Mathematical Functions with Formulas, Tenth Printing. Graphs, and Mathematical Tables, U.S. National Bureau of Standards, 1972.

[2] P.F. Byrd and M.D. Friedman. Handbook of Elliptic Integrals for Engineers and Scientists, 2nd Edition (revised), Springer-Verlag, 1971. MR.0277773 (43:3506)

[3] G. Eason, B. Noble and I.N. Sneddon. On certain integrals of Lipschitz-Hankel type involving products of Bessel functions, Philosophical. Transactions of the Royal Society of London, Series A, 247, pp. 529-551, 1955. MR0069961 (16:1107f)

[4] I.S. Gradshteyn and I. M. Ryzhik. Table of Integrals, Series and Products, Academic Press, 1980. MR 0582453(81g:33001)

[5] M.T. Hanson and I.W. Puja. The evaluation of certain infinite integrals involving products of Bessel functions: a correlation of formula, Quarterly of Applied Mathematics, 55 (3), pp. 505-524, 1997. MR 1466145 (98i:33005)

[6] A.E.H. Love. (1929). The stress produced in a semi-infinite solid by pressure on part of the boundary, Philosophical Transactions of the Royal Society of London, Series A, 228, pp. 377-420, 1929.

[7] F. Oberhettinger. Tables of Laplace transforms, Springer-Verlag, 1973. MR0352889 (50:5375) 